\title{
Doenças Negligenciadas - Malária: Testes com Chalconas e seus Derivados
}

\section{Wender A. Silva \& Larissa G. Maciel}

O desenvolvimento de novas chalconas e análogos vêm crescendo consideravelmente. A intenção desses estudos está em encontrar análogos com atividades comparáveis aos compostos utilizados comercialmente hoje, e que não desenvolva resistência do parasita ao medicamento. Essa pequena revisão visa mostrar alguns estudos realizados nos últimos anos com o objetivo de auxiliar em pesquisas futuras.

Palavras-chave: Chalconas, Ação Antimalarial, Plasmodium ssp.

The development of new chalcones and analogues has been growing considerably. The purpose of these studies is to find analogues with similar activities to compounds commercially used nowadays and that the parasite does not develop resistance to the drug. This review aims some studies carried out in recent years in order to assist in future research.

Keywords: Chalcones, Antimalarial Activity, Plasmodium ssp. 


\section{Introdução}

Ainda hoje, segundo estimativas cerca de 1 milhão de pessoas por ano vão a óbito no mundo devido a doenças negligenciadas ( são doenças endêmicas e tropicais onde a maior incidência ocorre em países subdesenvolvidos da África, Ásia e América Latina) como malária, dengue, esquistossomose, elefantíase, doença de Chagas. ${ }^{1}$ Um dos principais motivos do elevado numero de mortes se dá pela ausência de suporte no diagnóstico e no tratamento destes tipos de doenças, como a maior incidência se dá em países subdesenvolvidos ou em desenvolvimento para as grandes indústrias farmacêuticas não há apelo econômico no desenvolvimento de novos fármacos. Embora haja algumas ações governamentais e não governamentais no intuito de melhorar a relação custo-desenvolvimento, principalmente quando se analisa os impactos que essas doenças trazem no que tange gastos na saúde publica.

Em face ao exposto surgiu em meados de 2003, com intuito de pesquisar, desenvolver e disponibilizar medicamentos eficazes e financeiramente acessíveis para suprir deficiências no tratamento de doenças negligenciadas a Drugs for Neglected Diseases Initiative (DNDi) ${ }^{1}$, uma iniciativa dos médicos sem fronteiras e mais organizações públicas de pesquisa - Kenya Medical Research Institute, Indian Council of Medical Research, Malaysian Ministry of Health, Fundação Oswaldo Cruz, o Instituto Pasteur da França e mais o PNUD/Banco Mundial/Programa Especial de Pesquisa e Treinamento em Doenças Tropicais da Organização Mundial de Saúde (OMS/TDR). Estruturalmente a DNDi é uma organização sem fins lucrativos, cujo principal foco principal é até 2014 fornecer de seis a oito novos tratamentos que atendam às necessidades dos pacientes, e como objetivo secundário, a DNDi se esforça para utilizar e fortalecer a capacidade de pesquisa existente nos países endêmicos, além de tentar conscientizar o governo na sua responsabilidade no desenvolvimento e tratamento da população acometida por essas doenças.

\section{MALÁRIA}

A malária é uma doença infecciosa grave causada por um parasita, o Plasmódio as principais espécies são: Plasmodium falciparum, Plasmodium malariae, Plasmodium ovale e Plasmodium vivax ${ }^{2}$. Este último é transmitido ao ser humano através da picada de um mosquito fêmea, o vetor é o Anopheles (Anopheles sp), é encontrado freqüentemente nos países tropicais (Sudeste asiático, África, América Central) e subtropical (Brasil). A doença provocada pelo P.vivax é a mais comum, principalmente no Brasil, Figura 1, também conhecida como malária benigna, já a provocada pelo o malariae é a menos grave, no caso da infecção causada pela espécie ovale é típica da África. A contaminação oriunda do $P$. falciparum é mais danosa a saúde podendo levar a forma letal de malária. A malária também é conhecida como febre quartã, febre terçã benigna, febre terçã maligna, sezão, paludismo, maleita, tremedeira. Segundo a OMS hoje é responsável por até 300 milhões de casos clínicos por ano. ${ }^{3}$

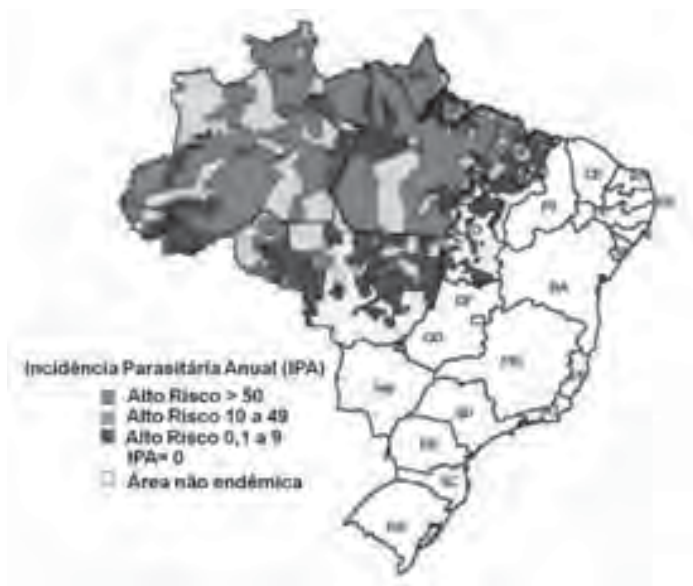

Figura 1. Áreas endêmicas de malaria no Brasil (adaptado referência 2).

De acordo com o manual de terapêutica da malária da funasa (Fundação Nacional de Saúde) ${ }^{2}$, o ciclo biológico do protozoário se dá da seguinte forma. "A infecção inicia-se quando esporozoítos infectantes são inoculados no homem pelo inseto vetor. Estas formas desaparecem da circulação sangüínea do indivíduo suscetível dentro de 30 a 60 minutos para alcançarem os hepatócitos, onde evoluem. Após invadir o hepatócito, os esporozoítos se diferenciam em trofozoítos pré-eritrocíticos. Estes se multiplicarão por reprodução assexuada do tipo esquizogonia, dando origem aos esquizontes teciduais e posteriormente a milhares de merozoítos que invadirão os eritrócitos. Esta primeira fase do ciclo é denominada exo-eritrocítica, pré-eritrocítica ou tecidual e, portanto, precede o ciclo sangüíneo do parasito (Figura 2). 
O desenvolvimento nas células do fígado requer aproximadamente uma semana para o $P$. falciparum e $P$. vivax e cerca de duas semanas para o $P$. malariae. Nas infecções por $P$. vivax, o mosquito vetor inocula populações geneticamente distintas de esporozoítos: algumas se desenvolvem rapidamente, enquanto outras ficam em estado de latência no hepatócito, sendo, por isso, denominadas hipnozoítos (do grego hypnos, sono). Estes hipnozoítos são responsáveis pelas recaídas da doença, que ocorrem após períodos variáveis de incubação, em geral dentro de seis meses para a maioria das cepas de $P$. vivax.”

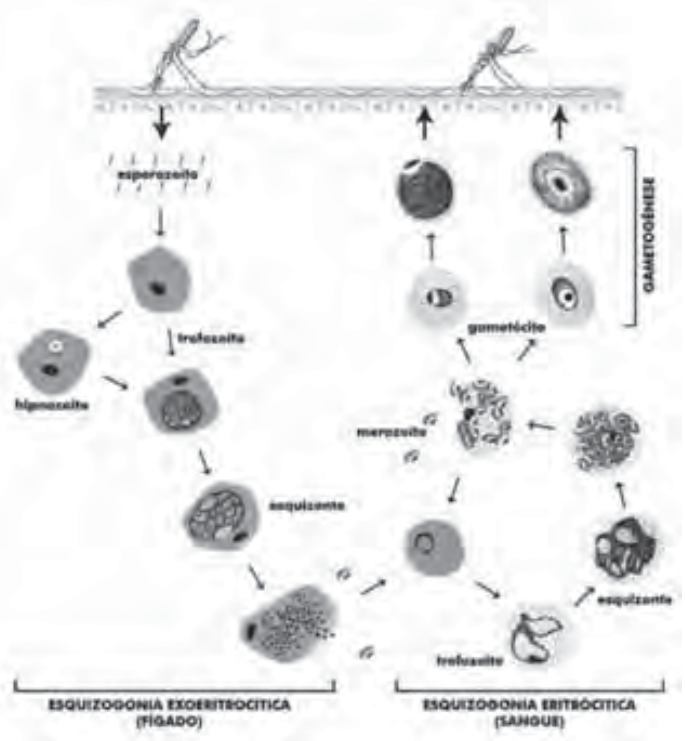

Figura 2. Ciclo biológico do protozoário.(adaptado referência 2).

\section{QUIMIOTERAPIA}

A falta de um tratamento efetivo contra as espécies de plasmódio mais reincidentes no Brasil ( $P$. vivax e $P$. falciparum) e a dificuldade em diferenciar clinicamente a infecção das espécies ou mesmo infecções simultâneas, torna a busca por quimioterápicos específicos mais atrativos, principalmente pelo fato da ação dos mesmos se dar em estágios bem definidos do ciclo biológico do protozoário. De modo geral o tratamento da malária visa principalmente cessar o estágio da esquizogonia sangüínea, estágio esse que indica manifestações clínicas da infecção². No entanto, pelos vários estágios descritos no ciclo biológico, torna-se também importante no tratamento a erradicação de formas latentes do parasita no ciclo tecidual (hipnozoítas), evitando assim as famosas recaídas. Durante o processo de desenvolvimento de novos protipos se deve implementar o uso de vacinas, no caso da malária essa tentativa data mais de 30 anos, porém seu uso ainda é um desafio uma vez que não age em todos os tipos de Plasmodium e sua ação deverá atuar em etapas específicas do ciclo de vida do protozoário ${ }^{4}$. Os principais fármacos utilizados estão listados abaixo e ilustrados na Figura 3:

- Quinolinometanóis naturais: quinina;

- Quinolinometanóis sintéticos: mefloquina;

- Fenantrenometanóis: halofantrina;

- 4-aminoquinolinas (cloroquina e amodiaquina);

-8-aminoquinolinas (primaquina);

- Lactonas sesquiterpênicas: derivados da artemisinina;

- Naftacenos: tetraciclinas (doxiciclina);

- Lincosaminas: clindamicina.

- Naftoquinonas (atovaquona).
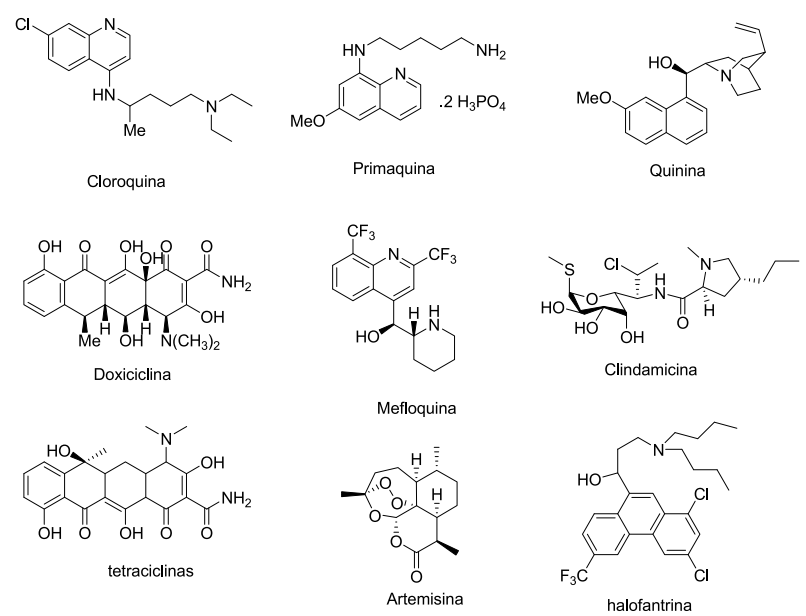

Figura3. Principaisquimioterápicosutilizadosnotratamentodamalária².

A quinina faz parte da família das quinolinas fazem parte dessa classe principalmente as 4-aminoquinolina, 8-aminoquinolinas e os quinolinometanóis. Essa classe é ativa frente às formas eritrocíticas de $P$. falciparum e $P$. vivax. Dessa classe a cloroquina é o mais eficaz, uma das substâncias mais utilizadas no combate e profilaxia da malária, mas infelizmente devido ao uso abusivo para esse fim levou à resistência 5 . 


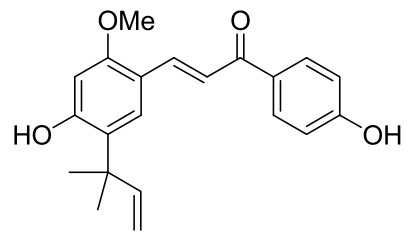

Figura 4. Estrutura da Licochalcona A.

Outra classe de fármacos bastante utilizados são os derivados do fenantreno, especificamente a halofantrina, apresenta alta eficiência contra a malária resistente à cloroquina e tem sido avaliado para o uso humano, apresentando com efeitos colaterais distúrbios gastrintestinais ${ }^{5}$.

O uso da mefloquina requer doses mínimas quando comparada a outros fármacos já utilizados no combate ao Plasmodium falciparum resistente à cloroquina ${ }^{5}$. Efeitos colaterais são relatados, os principais são lesões gastrintestinais, tonturas e efeitos psicológicos temporários, com isso seu uso não é recomendado a pessoas com histórico de epilepsia ou desordens psiquiátricas.

A artemisinina é um produto natural extraído da Artemisia annua, foi isolada na década de $70^{6}$, vários análogos já foram sintetizados com intuito de se avaliar suas atividades um desses são os derivados sesquiterpênicos que possuem uma ponte de peróxido sobre o ciclo de 7 membros do sistema tricíclico agindo como esquizonticidas sanguíneos, atuando também como gametocida, fazendo com que haja redução de formas resistentes?.

Muitos protótipos desenvolvidos nos últimos anos com a ação de inibir a atividade desse protozoário possuem relatos de resistência, deixando o tratamento ineficiente ${ }^{8}$. A busca emergencial de novos compostos com alta atividade antimalarial é justificada especialmente ao se gerar novas moléculas com ação igual ou superior a suas antecessoras que preferencialmente não gerem resistência durante o tratamento e estejam ancoradas em simplificações moleculares e na utilização de conceitos como a hibridização molecular. Partindo dessa premissa uma classe extremamente promissora, por possuir um amplo espectro de atividade e grande versatilidade sintética principalmente após o isolamento da Licochalcona A (Figura 4) e identificação de atividade frente à malária são as chalconas. Nos últimos anos houve um maior interesse em se investigar essa classe frente ao plasmodium sp com boa eficiência, muito provavelmente esse êxito se deve a adição de uma alguma espécie nucleofílica à ligação dupla da enona que é um excelente aceptor de Michael, posteriormente alguns resultados serão discutidos.

\section{CHALCONAS}

Por definição chalconas (1,3-diaril-2-propen-1-onas) são cetonas $\alpha, \beta$-insaturadas (Esquema 1), onde tanto o fragmento oriundo da carbonila quanto o fragmento olefínico estão ligados a anéis aromáticos, São precursoras de flavonóides e isoflavonóides que estão presentes na dieta humana, esta classe de compostos se torna de grande interesse apresentando vários estudos de isolamento, identificação e investigação de propriedades biológicas e farmacológicas. ${ }^{9}$

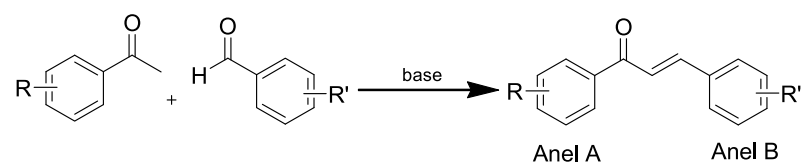

Esquema 1. Síntese de Chalconas

Algumas aplicações e propriedades das chalconas se destacam como, por exemplo: estudos de atividade multibiológicas, como antiinflamatórios ${ }^{10}$, anti-pirético ${ }^{11}$, efeitos anti-mutagênicos ${ }^{12}$, atividade antitumoral ${ }^{13}$, atividade contra Mycobacterium tuberculosis ${ }^{14}$, antifúngico ${ }^{15}$, antiviral ${ }^{16}$, inclusive com atividade anti-HIV ${ }^{17}$.

\section{Estudos de Casos}

Em estudos já realizados e descritos na literatura ${ }^{18}$ mostram de maneira geral a relação entre a estrutura e atividade das chalconas nos mecanismos de ação dos antimaláricos. Dentre os vários aspectos se destacam: (i) a influencia do anel B sobre a atividade está no tamanho e volume das substituições enquanto no anel A o aspecto primordial é a hidrofobicidade, (ii) Substituições com halogênios (principalmente cloro e flúor), no anel A não necessariamente irá incrementar a atividade antimalárica do composto, isso irá depender do tipo de substituição no anel $\mathrm{B}$, para que haja sinergismo entre os grupos substituintes nos anéis, (iii) fatores estéricos e hidrofóbicos, poderá ser uma limitação tanto que o tamanho da molécula ao longo do eixo $\mathrm{X}$ das chalconas é relativamente pequeno, da mesma maneira que o crescimento da molécula no eixo y deve ser 
limitada para que não haja decréscimo nas interações e por conseguinte na atividade, (iv) alta densidade de elétrons nos $\mathrm{C}_{3}, \mathrm{C}_{4}$ do anel $\mathrm{B}$ poderá interferir positivamente na atividade do composto em estudo, (v) a condição de existir grupos aceptores de ligação de hidrogênio no $\mathrm{C}_{3} \mathrm{e}$ $\mathrm{C}_{4}$ do anel $\mathrm{B}$, para que se direcione a geração de ligações de hidrogênio mais eficientes, um bom exemplo é a licochalcona A (Figura 4).

A licochalcona A é isolada a partir de extratos de raízes da Glycyrrhizia glabra ${ }^{19}$ e tem sido relatada como uma molécula promissora com alta atividade antimalárica, a partir de uma varredura in vitro frente à cepa sensível a cloroquina (3D7) e a cepa resistente a cloroquina (DD2) isolado a partir do Plasmodium falciparum. ${ }^{20} \mathrm{~A}$ eficiência desta classe de compostos também foi confirmada frente a outras espécies de Plasmodium (P.yoelii, com ocorrência na África) testados em camundongos. Com isso proporcionou interesse em projetar e sintetizar novos tipos chalconas e bischalconas e analisar a influência de grupos substituintes bem como a importância de seus posicionamentos com o objetivo de identificar potenciais alvos antimaláricos.

O estudo realizado por Wanare et.al ${ }^{21}$ se baseou no uso de hibridização molecular na síntese de $\alpha$-piranochalconas (derivados de chalconas e também dos flavonóides). Dos compostos sintetizados e analisados, cinco obtiveram resultados significativos atividades ( $\mathrm{IC}_{50}<10 \mu \mathrm{g} / \mathrm{mL}$ ), melhor representados no Esquema 2. De acordo como os autores, uma possível justificativa para alta atividade dessas moléculas pode ser a hidrofobicidade e as interações dos substituintes no sitio ativo da enzima falcipaína Figura 5. A alta atividade dos compostos trimetoxila substituídos está de acordo com a influência da hidrofobicidade e volume estérico no anel A. Entre os compostos dissubstituídos, os compostos mono-hidroxilado são mais ativo do que os derivados tipo dimetóxi substituído.

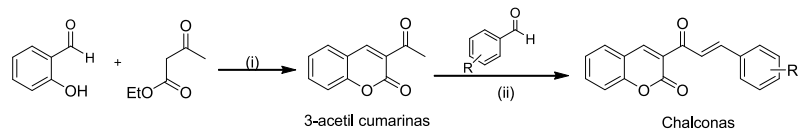

(i) piperidina, etanol, t.a; (ii) silica, ácido sulfúrico, sem solvente.

Esquema 2. Síntese da $\alpha$ - piranochalconas obtida a partir da acetil cumarina

No trabalho de Dominguez et al..$^{22}$ os estudos foram baseados em sistemas derivados de vinil sulfonas (Figura 6), dos compostos sintetizados nove compostos entre mono e dissubstituídos tiveram $\mathrm{IC}_{50}$ abaixo de $10 \mu \mathrm{g} /$ $\mathrm{mL}$ perante o Plasmodium Falciparum. Os tipos de substituintes utilizados nos compostos com os valores dos respectivos IC $_{50}$ estão dispostos na Tabela 1.

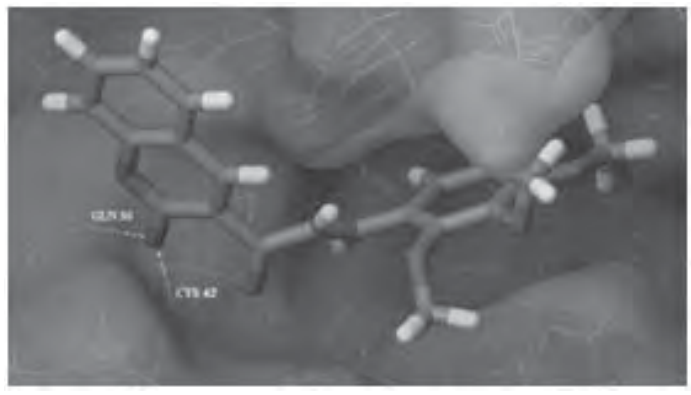

Figura 5. Docking da piranochalcona com resíduo cys 42 do sitio ativo da enzima falcipaína. ${ }^{21}$

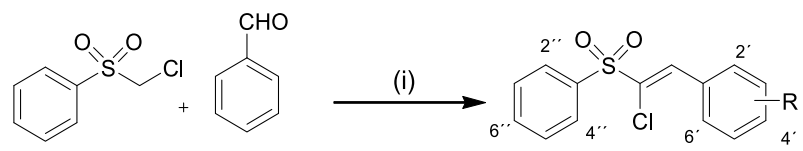

Figura 6. Reagentes e produto da reação de síntese do cloro-vinil sulfona.

A partir dos resultados obtidos, os autores discutem sobre a importância dos grupos 3,4-metilenodioxila localizado no anel aromático oriundo do aldeído, bem como o grupo 2-cloroquinolinil também oriundo do fragmento do aldeído, tem grande influência na atividade contra o P. Falciparum, podendo ocorrer alguma interação entre a chalcona e a enzima por mecanismos ainda não identificados. Compostos com a presença de substituintes com cloro, nitro ou naftila em diferentes posições dos anéis aromáticos apresentam atividade antimalárica, porém nenhuma dessas modificações estruturais aproximou ao nível de atividade compostos da entrada 3 e 6, mostrados na Tabela 1. Posteriormente será sintetizada uma segunda geração de compostos, baseado em SAR com objetivo de diminuir a concentração do $\mathrm{IC}_{50}$ para ordem de $\mathrm{nM}$, utilizando como fragmentos precursores os das entradas 3 e 7 .

Segundo Dominguéz et.al ${ }^{23}$ a maioria das sulfonamidas-chalconas dissubstituídas ou tri-substituída (Figura 7) com grupos metoxila possuem atividades significativas na inibição da $\beta$-hematita. Foi observado 
também que compostos com substituições (Cl, F, $\mathrm{CH}_{3}$ ou $\mathrm{OCH}_{3}$ ) não aumentam a atividade sulfonamidas-chalconas quando comparado, com os compostos di e trimetoxila substituídos. Uma informação importante é que compostos que não são fortemente básicos não vão possuir uma boa atividade antimalárica, uma vez que o parasita após se ligar as hemácias se armazenam em vacúolos digestivos ácidos, no qual a hemozoína (hidrolise da hemoglobina para produção do pigmento malárico), é formada.

Tabela 1. Substituintes da cloro-vinilsulfona e suas concentrações capazes de inibir 50\% de uma determinada atividade (adaptada) ${ }^{22}$.

\begin{tabular}{|c|c|c|}
\hline Entrada & Substituintes & IC $_{50}(\mu \mathrm{M})$ \\
\hline 1 & 2,4- $\mathrm{diCl}_{2}$ & 0.96 \\
\hline 2 & 4-OMe & 0.47 \\
\hline 3 & $3,4-\mathrm{OCH}_{2} \mathrm{O}-$ & 0.053 \\
\hline 4 & 4-Cl & 0.083 \\
\hline 5 & $3,4-\mathrm{diCl}_{2}$ & 0.059 \\
\hline 6 & 2-(1-naftil) & 0.058 \\
\hline 7 & 2-Cl-3-quinolil & 0.025 \\
\hline 8 & $4-\mathrm{NO}_{2}$ & 0.073 \\
\hline 9 & 1-naftil & 0.075 \\
\hline
\end{tabular}
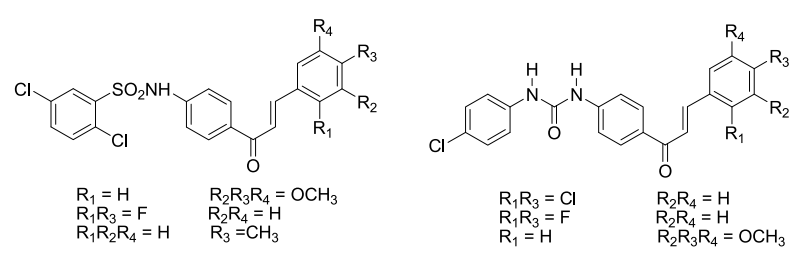

Figura 7. Chalconas sulfonamidas substituídas.

Outra classe de compostos estudada são as bischalconas, Figura 8, relatadas por Ram et.al ${ }^{24}$ e Xue et.al ${ }^{25} \mathrm{com}$ atividade antimalárica in vivo contra cepas sensíveis e resistentes a cloroquina em camundongos. Esses estudos mostram que o posicionamento do grupo oxigenado (espaçadores), que fazem a ligação entre os anéis é importante na determinação do perfil de atividade. De modo geral bischalconas, com o substituinte metoxila nas posições 3,4 e meta apresentam maior atividade que compostos com substituintes 2,4-dimetoxila. Os espaçadores também possuem papel importante na atividade, compostos com três grupos metileno contribuem mais do que aqueles com quatro ou seis, uma possível reposta para essa diferenciação se dá pelo distanciamento entre as chalconas, fazendo com que a interação com o sitio ativo se torne mais difícil, por questões estéricas e eletrônicas.

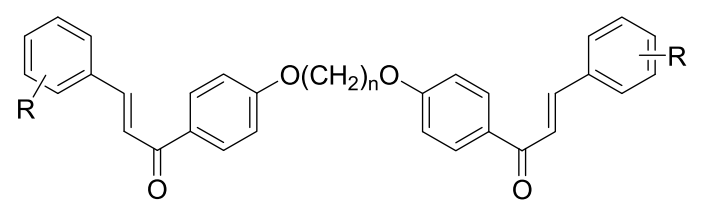

$\mathrm{n}=1,3,4,6 \mathrm{R}=3,4-\left(\mathrm{OCH}_{3}\right)_{2}$ ou $3-\mathrm{OCH}_{3}$ ou $2,4-\left(\mathrm{OCH}_{3}\right)_{2}$

Figura 8. Bischalconas substituídas

Outra chalcona estudada foi a Crotaorixina ${ }^{26}$ que foi isolada das folhas da Orixensis Crotalaria. Esse composto exibiu $100 \%$ de inibição para a cepa NF54 (P.falciparum), o medicagenina que é diprenilado foi isolado das raízes e apresentou também excelente inibição frente à cepa NF-54. Já as demais chalconas isoladas apresentaram atividades menores quando comparadas aos compostos mostrados na Figura 9. A crotaorixina quando prenilada nas posições para, teve sua atividade aumentada mostrando a importância do grupo prenil nesse sistema.
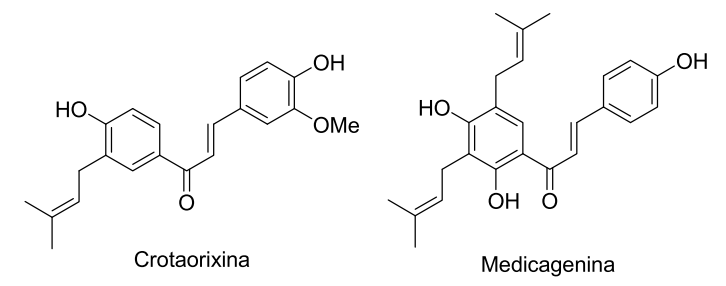

Figura 9. Chalconas isoladas de diferentes espécies de Crotalaria.

\section{Considerações Finais}

Essa breve revisão visa mostrar alguns aspectos importantes relacionados à malária, os principais quimioterápicos em uso bem como o estudo de potenciais protótipos no combate a esta doença negligenciada que acomete milhões de pessoas por ano em países menos favorecidos.

O protozoário que causa a malária possui um ciclo de vida bem definido, podendo infectar tanto células hepáticas quanto sanguíneas, o que dificulta a ação de fármacos cuja ação não é tão seletiva. Devido essas características o desenvolvimento de vacinas contra a 
malária se torna um tema latente no meio científico. Com esse trabalho visamos mostrar novas possibilidades de tratamento utilizando uma classe versátil de moléculas que já atuam e poderão atuar de forma mais eficiente como quimioterápicos contra a malária. Nosso grupo de pesquisa (LaPSCA-Laboratório de Planejamento e Síntese de Compostos Ativos) trabalha de forma incessante no aprimoramento estrutural baseados em ensaios e estudos de QSAR para síntese de novas chalconas, e com isso esperamos estar auxiliando e estimulando a pesquisa cientifica em nossa região.

\section{Referências}

1. Pontes, F.; Revista Inovação em Pauta, 2009, 6, 69.

2. Manual de Terapêutica da Malária, Brasília: Ministério da Saúde. Fundação Nacional de Saúde. 2001.

3. www.sucen.sp.gov.br acessado em 31/10/2011

4. Nussensweig, R.S. Nature, 1967, 216, 160.

5. Gregson, A.; Plowe, C. V.; Pharmacological Rev. 2005, 57, 117.

6. Wright, C. W.; J. Ethnopharmacol. 2005, 100, 67.

7. Hyde, J. E.; Microbes and Infection 2002, 4, 165.

8. Padmanabhan, G.; Rangarajan, P.N.; Biochem. Biophys. Res. Commun. 2000; 268, 665.

9. a) Iwashina, T., Kitajima, J. Phytochemistry 2000, 55, 971.; b) Silva, W. A., Gatto, C. C.; Oliveira G. R..; Acta Cryst. 2011. E67, o2210,c) Vencato, I.;Andrade, C.K.Z., Silva, W.A.; Lariucci,C.; Acta Cryst. 2006. E62, o1033. d) Lawrence, N. J.; Rennison, D.; McGown, A. T.; Ducki, S.; Gul, L. A.; Hadfield, J. A.; Khan, N.; J. Comb. Chem. 2001, 3, 421.

10. Hsieh, H. K.; Lee, T. H.; Wang, J. P.; Way, J. J.; Lin, C. N.; Pharmacol. Res. 1998, 15, 39.

11. Satyanarayana, K.; Rao, M. N. A.; Indian Drugs 1993, 30, 313.

12. Torigoo, T.; Arisawa, M.; Iloch, S.; Fujiu, M.; Mayuyama, H.; Biochem. Biophys. Res. Commun. 1983, 112, 833.

13. a) Harborne, J. B.; Williams, C. A. Phytochemistry. 2000, 55, 481. (b) Houghton, P. J. Stud. Nat. Prod. Chem. 2000, 21, 123.

14. Lall, N.; Hussein, A. A.; Meyer, J. M. M.; Fitoterapia, 2006, 77, 230.
15. Ivanova, A.; Batovska, D.; Engi, H.; Parushev, S.; Ocsovszki, I.; Kostova, I.; Molnar, J.; Eur.J. Med. Chem. 2006, 22, 379.

16. Kiat, T.S. Bioorg. Med. Chem. Lett. 2006, 16, 3337.

17. Cheenpracha, S. Bioorg. Med. Chem. Lett, 2006, 14, 1710.

18. a) Li, R.; Chen, X.; Gong, B.; Selzer, P. M.; Li, Z.; Davidson, E.; Kurzban, G.; Miller, R. E.; Nuzum, E. O.; McKerrow, J. H.; Fletterick, R. J.; Gillmor, S. A.; Craik, C. S.;Kuntz, I. D.; Cohen, F. E.; Kenyon, G. L. Bioorg. Med. Chem. 1996, 4, 1421. (b) Liu, M.; Wilairat, P.; Go, M. L. J. Med. Chem. 2001, 44, 4443; (c) Liu, M.; Wilairat, P.; Croft, S. L.; Tan, A. L. C.; Go, M. L. Bioorg. Med. Chem. 2003, 11, 2729; (d) Xue, C. X.; Cui, S. Y.; Liu, M. C.; Hu, Z. D.; Fan, B. T. Eur. J. Med. Chem. 2004, 39, 745.

19. Chen, M.; Theander, T. G.; Christensen, S.B.; Hviid, L.; Zhai, L.; Kharazmi, A.; Antimicrob. Agents Chemother. 1994, 38, 1470.

20. Liu, M.; Wilairat, P.; Go, M-L.; Liu, M.; J. Med. Chem. 2001, 44 , 4443.

21. Wanare, G.; Aher,R.; Kawathekar, N.; Ranjan,R.; Kaushik, N. K.; Sahal, D.; Bioorg. Med. Chem. Lett. 2010, 20, 4675.

22. Dominguez, J. N.; Leon, N.; Rodrigues, J.; Gamboa de Dominguez, N.; Gut,J.; Rosenthal, P. J.; Eur. J. Med. Chem, 2009, 44, 1457.

23. Domínguez, J. N.; Leon, C.; Rodrigues, J.; Gamboa de Dominguez, N.; Gut, J.; Rosenthal, P.J.; Farmaco, 2005, 60, 307.

24. Ram, V. J.; Saxena, A. S.; Srivastava, S.; Chandra, S.; Bioorg. Med. Chem. Lett. 2000, 10, 2159.

25. Xue, C. X.; Cui,S.Y.; Liu, M. C.; Hu. Z.D.; Fan, B.T.; Eur. J. Med. Chem. 2004, 39, 745.

26. Narender, T.; Tanvir, K.; Srinivasa, M. R.; Srivastava, K.; Puri, S. K.; Bioorg. Med. Chem. Lett. 2005, 15, 2453.

\section{Wender A. Silva* \& Larissa G. Maciel}

LaPSCA- Laboratório de Planejamento e Síntese de Compostos Ativos. Instituto de Química, Universidade de Brasília, Campus Darcy Ribeiro, Caixa Postal. 4478, CEP 70910-970, Brasília/DF, Brasil.

*e-mail: wender@unb.br 